\title{
Efficient Iris Pattern Recognition Method by using Adaptive Hamming Distance and 1D Log-Gabor Filter
}

\author{
Rachida Tobji ${ }^{1}$, Wu Di ${ }^{2} *$, Naeem Ayoub ${ }^{3}$, Samia Haouassi ${ }^{4}$ \\ School of Computer Science and Technology \\ Dalian University of Technology, Dalian 116000, China
}

\begin{abstract}
Iris recognition is one of the highly reliable security methods as compared to the other bio-metric security techniques. The iris is an internal organ whose texture is randomly determined during embryonic gestation and is amenable with a computerized machine vision system for the remote examination. Previously, researchers utilized different approaches like Hamming Distance in their iris recognition algorithms. In this paper, we propose a new method to improve the performance of the iris recognition matching system. Firstly, 1D Log-Gabor Filter is used to encode the unique features of iris into the binary template. The efficiency of the algorithm can be increased by taking into account the coincidence fragile bit's location with 1D Log-Gabor filter. Secondly, Adaptive Hamming Distance is used to examine the affinity of two templates. The main steps of proposed iris recognition algorithm are segmentation by using the Hough's circular transformation method, normalization by Daugman's rubber sheet model that provides a high percentage of accuracy, feature encoding and matching. Simulation studies are made to test the validity of the proposed algorithm. The results obtained ensure the superior performance of our algorithm against several state-of-the-art iris matching algorithms. Experiments are performed on the CASIA V1.0 iris database, the success of the proposed method with a genuine acceptance rate is $\mathbf{9 9 . 9 2 \%}$.
\end{abstract}

Keywords-Iris recognition; bio-metric; Hamming Distance; iris recognition matching; Adaptive Hamming Distance; 1D Log-Gabor Filter; segmentation; normalization; feature encoding; genuine acceptance rate

\section{INTRODUCTION}

Bio-metric security techniques are comprehensive techniques that establish a secure identity of a person by measuring one or more types of physical characteristics. Bio-metric based techniques are currently favored by a fashion phenomenon, mainly film and television reported in [1]. Thus, it is not uncommon to see retinal scanners with beautiful red lasers, readers fingerprints with very nice flashing lights, etc. All this becoming the ultimate in access control technology. There are some bio-metric techniques that have been used from the last few decades but they have some limitations. In this study we focus on iris bio-metric security technique which is the most reliable and accurate among all bio-metric traits at present. It has been proved that the probability of finding two identical irises is less than the reverse of the number of humans who have lived on earth. Fig. 1 shows structure of human eye.

Iris recognition is based on interpretation and comparison information between two irises of the same person, belonging to two individuals who may be the same person or not. In

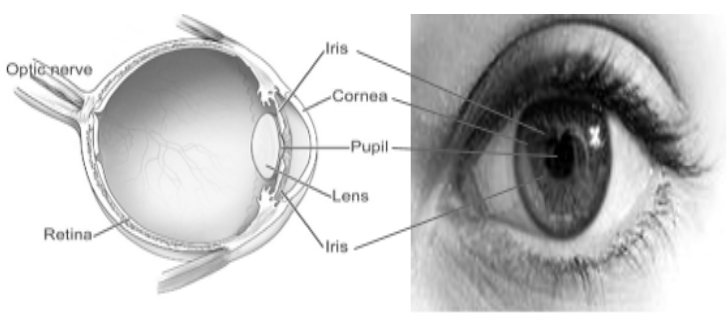

Fig. 1. Structure of human eye.

the first case, there must be authentication, but authentication should not be performed in the second case. Selection of iris images may have different size and orientation between several photos, it is therefore necessary to use information that is independent of the parameters of the image. It is necessary to eliminate the pupil, the white of the eye, perhaps the reflections and the area of the eyelid in the case of significant encroachment on the iris. This operation will make subsequent processing more reliable, as compares iris information only, and in addition gain memory occupancy when saving code vectors.

In an eye, iris is the colored area between the pupil and the white of eye. The iris has diameter smaller than a hair which consists of a network of the fine tubes. Iris formation in the human eye begins in the third month and the structure of the distinctive elements finishes in the eighth month of gestation. The pigmentation process continues in the first years of birth. The formation of the iris is chaotic which generates patterns with high variability.

There are two main stages of iris recognition, first, the features of are recorded in the enrollment process. Second, in matching process, the authentication system attempts the confirmation of an individual's identity by comparing a submitted sample to previously enrolled templates.

The remaining sections of this paper are organized as follows: Background is described in section 2. In section 3 related work is briefly discussed regarding iris bio-metric systems based on Hamming Distance with feature encoding schemes adopted by different researchers. Section 4 proposed method and provides a detailed description of iris recognition system components with pre-processing i.e., image acquisition, segmentation and normalization, feature extraction and encoding, matching. Experimental results and discussion are presented in section 5. Finally, conclusion is made in section 
6.

\section{BACKGROUND}

The American ophthalmologist Frank Burch was the first person who had officially realized the possibilities of the texture of the iris as an identification tool. He proposed this method in a lecture that was delivered in American Academy of Ophthalmology in 1936. Before that, the iris was mainly considered only for its color. Burch's idea has been reproduced in the ophthalmology manuals, but there was very little research done, due to particular lack in the field of engineering [2]. Professor John Daugman developed a mathematical approach to the analysis of patterns of the iris. The collaboration between Daugman, Sarin and Flom was resulted in a functional prototype patented in 1994. This system has been greatly improved. Several companies used licenses of iris recognition algorithms in their applications. The patent is now the property of the company iridian. Daugman's work was based on the non-orthogonal Gabor complex wavelets, the complex-valued filters were applied to the texture of the twodimensional iris and the phase information was extracted to form the signature [3], [4]. Wildes [5] presented a system based on a pyramidal representation multi-resolution iris textures. The idea behind the image pyramid is as follows: an image can be represented as a sub-sampled approximation and one or more images residuals at different resolutions. In Wildes's system four sub-images were used from a multi-resolution pyramid as a signature and a standardized correlation was measured for classification. Boles et al. [6] proposed an algorithm based on "Zero-Crossings" of a one-dimensional orthogonal wavelet transform. First, the one-dimensional signal was transformed and acquired by recording the gray scale values of one or several concentric circles of the iris. Then the radius of the circle was normalized with respect to the radius of the iris, ensuring that the same points of the texture of the iris sampled according to size of the iris in the picture. Zhu et al. [7] have done comparative studies between wavelets non-orthogonal Gabor and orthogonal wavelet of Daubechies of order 4 ("db4"). In this method, Gabor wavelets multidirectional and different frequencies were used to separate the texture of the iris in different frequency sub-bands. The average and standard deviation were used as a signature. In the case of wavelets, the mean and the standard deviation of the sub-bands were generated from a transformation on five levels and used as signature. The result was very much in favor of the wavelet analysis of Gabor. Lim et al [8] used an orthogonal wavelet transform with a simple Haar Wavelet. Four levels of transformations were calculated first and the signature was composed based on the coefficients of the details diagonals at the lowest scale. Each average value of the diagonal detailed the coefficients to the other three subbands at higher. The signature coefficients were all of zero mean and truncated to only one bit as a function of their sign. Tisse et al. [9] used the Hilbert transform for iris detection. In which frequency emergent and the instantaneous phases (combination of the original signal and the Hilbert transform) were used to generate the signature. Then Hamming Distance was calculated to compare two signatures. Rydgren et al. [10] used a wavelet packet transform on three levels and applied it to the unrolled images. The sub images in the wavelet packet tree which holds information about both frequency and location were calculated as the suitable candidates for iris signatures. An energy measure was used to identify the particular packets that carries discriminating information about the iris texture. Noh et al. [11] used a method called multiresolution ICA by calculating the value in average gray levels as a function. They construct a one-dimensional iris signature which served as input to the multi-resolution ICA algorithm. In addition, a presentation of some current methods as well as a very recent global study that covers development history and current state-of-the art for understanding the recognition of the iris was also presented in literature [12]. Nabti and Bouridane [13] introduced a new approach to Multi-Scale contour detection for iris segmentation. Lionel [14] proposed a new iris recognition system to improve safety by using a technique of portable object. He studied a human-machine based on gesture recognition. In a first portion, he evaluated grid photo sensors from modeling up to the characterization of pixels and then tested the vehicle in CMOS technology that allowed them to compare 24 pixels to photo grid. He also addressed research on the integration of video stabilization on a sensor of images, key points on portable objects and on near infrared filtering. Wang et al. [15] introduced an eyelash detection method, in which a combination of Expectation Maximization (EM) and Gaussian Mixture Model(GMM) was used to extract the eyelash and eyelids information. Wang et al. [16] used random transform with polynomial curve fitting and least squares fitting to detect the eyelid and eyelashes. They used diagonal gradient and thresholding method to get the information of eyelashes .

Several iris recognition techniques have been developed by employing the traditional iris recognition systems [17], [18], [19], [20], feature value extraction [3], [21], [22], [23], [24] and iris matching [25], [26]. Most of state-of-the-art methods used simple binarization based on the sign of feature values [3],[21], [22],[27], [28].

\section{RELATED WORK}

In iris localization methods, an integro-differential operator was used by Daugman [21], which is as follows:

$$
\max _{\left(r, x_{0}, y_{0}\right)}\left|G_{\sigma}(r) * \frac{\partial}{\partial_{r}} \oint_{r, x_{0}, y_{0}} \frac{I(x, y)}{2 \Pi r} d s\right|
$$

Where $I(x, y)$ is an image containing an eye. By equation (1), maximum angular integral of radial derivative over the image domain, the outer (limbus) boundary of the iris and pupillary boundary location can be realized. Firstly, the outer limbus boundary location can be estimated, to avoid the eyelids occlusion, the limit of angular arc of contour is bounded in range to $90^{\circ}$ centered on the horizontal meridian. Secondly, a finer convolution scale $\sigma$ find pupillary boundary. Wildes [5] proposed a method to estimat the iris boundaries by adopting the hough transform. Masek [29] also used hough transform method for iris boundary estimation. These two methods can efficiently estimate the iris boundaries. In [12] and [30], a different method was introduced by using the active contours to find the non-circular shape of the iris which gives better representation of circle. This method does not perform well in some cases because if the edges details are weak then the desired iris boundary can not be evaluated according to the iris pattern. A threshold method has also been adopted by Somnath 


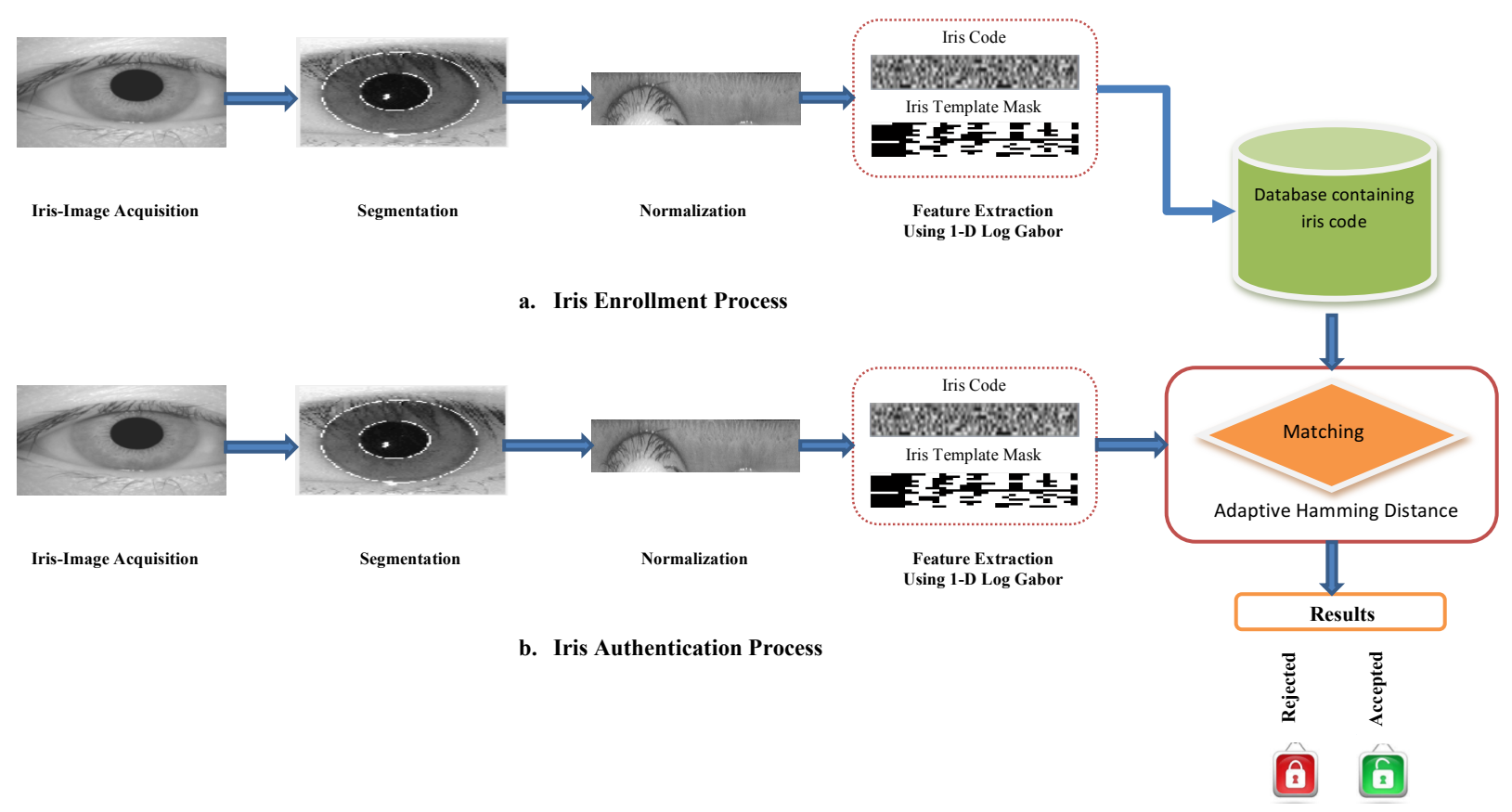

Fig. 2. Flow diagram proposed iris biometric system.

et al. [31] to detect boundary of the pupil in the eye image areas as it is darker than the other. Different state-of-the art techniques used fourier methods to account eyelid occlusions and iris segmentation. Avila [32] presented a similar type of system, in which he used a zero-crossing discrete dyadic wavelet transform which showed a high level of accuracy. Li Ma et al. [33] proposed to use circular symmetry filters to capture local-texture information of the iris. Then they utilized this information to construct feature vector the fixed length. For instance, Tisse et al. [34] proposed new approach for extraction and localization of iris by implementing $C$ language and tested individually the performance of the different processing. Rai et al. [35] used Support Vector Machine (SVM), Hamming Distance, parabola detection and trimmed median filter to detect and remove the eyelid, and eyelashes from the iris image. Soliman et al. [36] used a coarse-to-fine algorithm to address the computational cost problem and the integrodifferential operator to get information aboutpupil centers and radii. In [37] Dehkordi et al. used Adaptive Hamming Distance to improve the performance of iris code matching stage. The expanding and adjoining behavior of Hamming subsets to the right or left neighboring bits increased the accuracy of Hamming distance computation.

\section{PRoposed Method}

In this paper, we prpose an approach for iris pattern recognition by Adaptive Hamming Distance with improving the speed and accuracy of the process. we use a statistical method to account eyelashes presented in litirature [12] and 1D Log-Gabor Filter features to uniquely identify iris. We have studied various well known algorithms for iris recognition [29],[32], [33], [34], [35], [36], [37] and compared the results with state-of-the art algorithms. Fig. 2 shows flow diagram of iris biometric system which is described in detail in the following subsection.

\section{A. Iris Recognition System}

The proposed iris recognition system based on the five main steps. In the first step, the person's eye enrollment is performed. In the second step, iris is segmented from the image to locate and study the iris pattern. In the third step, we normalize and scaled the iris pattern to a predefined size. In forth step, we extract the features of iris code from filtered iris details, obtained from generated iris templates. In the fifth step, we compute the similarity score of two iris codes by matching them with Adaptive Hamming Distance scheme. For generating the base templates, we use the same methods as introduced in [29] with the Adaptive Hamming Distance for handling the images of CASIA V1.0 [38]. The same methodology adopted by Daugman [21] in the matching by metric employed and to extract textural information of iris image. Fig. 3 shows the steps of an iris recognition system.

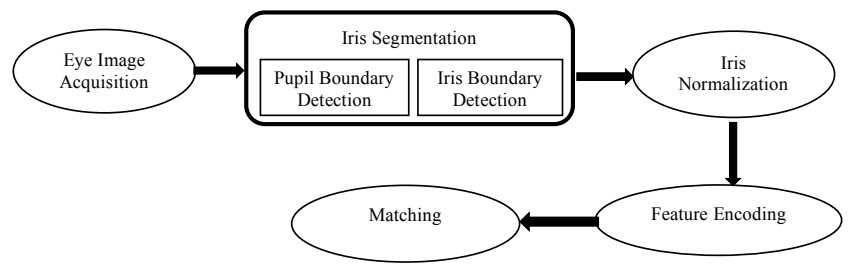

Fig. 3. Typical main steps of an iris recognition system.

The detailed mathodology is given in the subsubsections below.

1) Image Acquisition: Iris image acquisition is an important step in the recognition system. In this step images are acquired using CCD camera. Images having good resolution and sharpness need to maintain with adequate intensity. For 
the purpose of fair evaluation of our proposed algorithm, we use CASIA iris image database (version 1.0).

2) Segmentation and Normalization : For the purpose of segmentation, we isolate the zone of the iris of the eye (Fig. 4-a), then horizontal direction and vertical direction are estimation for segmentation. We use the description of the function which locates the center of the image. Once we get both directions, it becomes easy to detect the center (Fig. 4b). To detect the centers, Hough transform is applied to the circular forms to detect the border of iris / pupil. For effective and precise circle, first Hough transform was executed for the border of iris / sclera then for the border of iris / pupil (Fig. 4c). Fig. 4 shows steps of Segmentation.

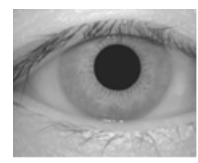

(a) Inis base.

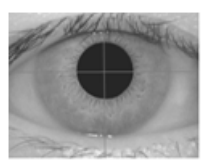

(b) Horizontal and Vertical Diameters

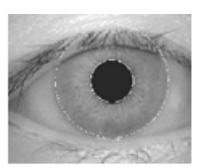

(c) Located Iris.
Fig. 4. example of steps of Segmentation: (a) Iris base, (b) Horizontal and Vertical Diameters, (c) Located Iris.

Once the iris region is successfully segmented, we normalize the iris segmented area by transforming the representation space of the iris with rectangular space to get fixed dimensions for authentication of the data that has the same dimension as shown in Fig. 5.
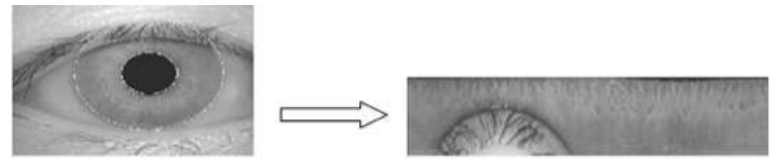

Fig. 5. Normalization of the localized iris.

In the normalization process, we unwrap the iris image and convert it to its polar equivalent by using Daugman's Rubber sheet model. The points on the Cartesian scale to the polar scale of center of the pupil are converted through a remapping formula. The remapping of iris image $\mathrm{I}(\mathrm{x}, \mathrm{y})$ from raw Cartesian coordinates to polar coordinates $(r, \theta)$ can be calculated as follows

$$
I(x(r, \theta), y(r, \theta)) \longrightarrow I(r, \theta)
$$

Where $\mathrm{r}$ is on the interval $[\theta, 1]$ and $\theta$ is angle $[\theta, 2 \pi]$, with:

$$
\begin{aligned}
& x(r, \theta)=(1-r) x_{p}(\theta)+r x_{l}(\theta) \\
& y(r, \theta)=(1-r) y_{p}(\theta)+r y_{l}(\theta)
\end{aligned}
$$

Where $x_{p}(\theta), y_{p}(\theta)$ and $x_{l}(\theta), y_{l}(\theta)$ are the coordinates of the pupil and iris boundaries along the direction $\theta$.

Daugman's Rubber sheet model is shown in Fig. 6.

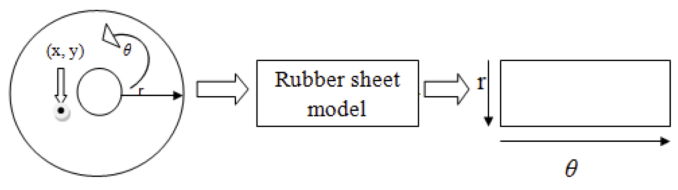

Fig. 6. Daugman's Rubber sheet model for the normalization of the iris.

3) Feature Extraction and Encoding: In this step, a template that represent the iris pattern information is generated by using a 1D log-Gabor filter. The error between the two different images occur when the pixels' intensity of two different iris images directly compared. To diminish this difficulty, 1D LogGabor filter is used same as [39], [40]. 1D Log-Gabor filter can be calculated by equation (5) as follows :

$$
G_{(X, Y)}=e^{-\frac{\log \left(\frac{\text { radius }}{f o}\right)^{2}}{2 * \log (\text { sigmaOnf })^{2}}}
$$

To extract and encode image texture information, the filters are multiplied by the raw image pixel data and coefficients are generated by integrating them over their supported domain. A noise mask is then generated with the feature template to mark the corrupted bits in the template. Fig. 7 shows feature encoding of iris.

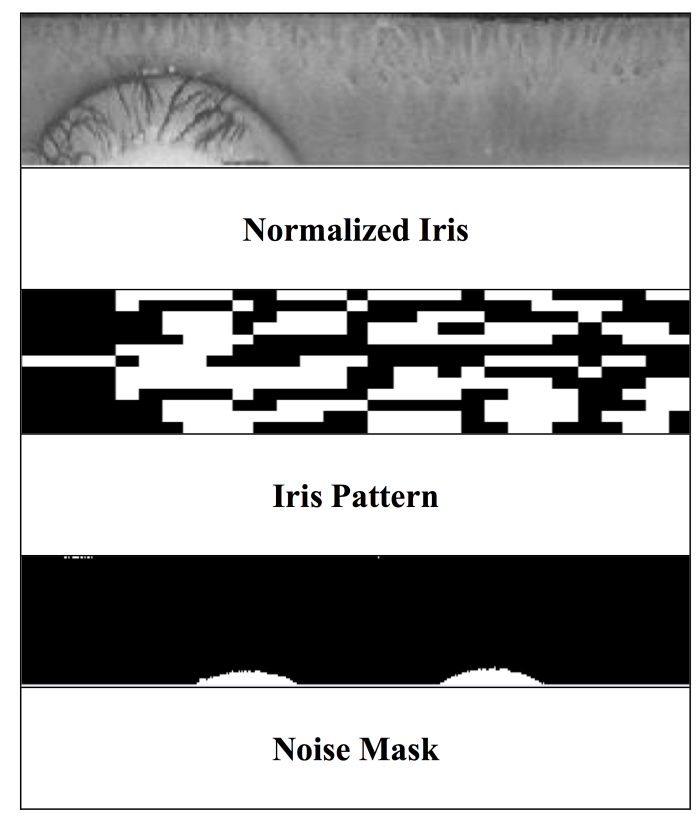

Fig. 7. Feature encoding.

4) Matching: Matching step consists of all the previous steps that are performed through out the enrollment of the encoded iris template. By extracting the binary form of the encrypted bit pattern, a simple boolean "XOR" operation can be used to match the stored encrypted bit patterns [41]. The dissimilarity measure is computed between any two iris bit patterns by utilizing the Adaptive Hamming Distance. The 
Hamming distance can be defined as follows

$$
H D=\frac{1}{N} \sum_{j=1}^{N} X_{j} \oplus Y_{j}
$$

Where, $\mathrm{N}$ represents the total number of the bits per bit pattern. HD represents the fractional measure of dissimilarity and 0 will indicate the perfect match. The Adaptive Hamming Distance can be calculated by the following formula

$$
H D=\frac{\left\|\left(\operatorname{Code}_{A} \cap \operatorname{Mask}_{A}\right) \oplus\left(\operatorname{Code}_{B} \cap \operatorname{Mask}_{B}\right)\right\|}{\left\|\operatorname{Mask}_{A} \cup \operatorname{Mask}_{B}\right\|}
$$

In this equation (7), $\operatorname{Code}_{A}$ and $\mathrm{Mask}_{A}$ represent the iris code of unidentified individual $A$. Code $_{B}$ and $M a s k_{B}$ represents kits of template $B$ of bitwise iris code and mask vector, which contains $20 * 480=9600$ template bits and $20 * 480=9600$ mask bits, respectively, that are calculated by using "XOR" and "AND" boolean operators. $\|$ Mask $_{A} \cup$ Mask $_{B} \|$ denotes the total number of comparisons. Algorithm 1 shows the general steps of matching method.

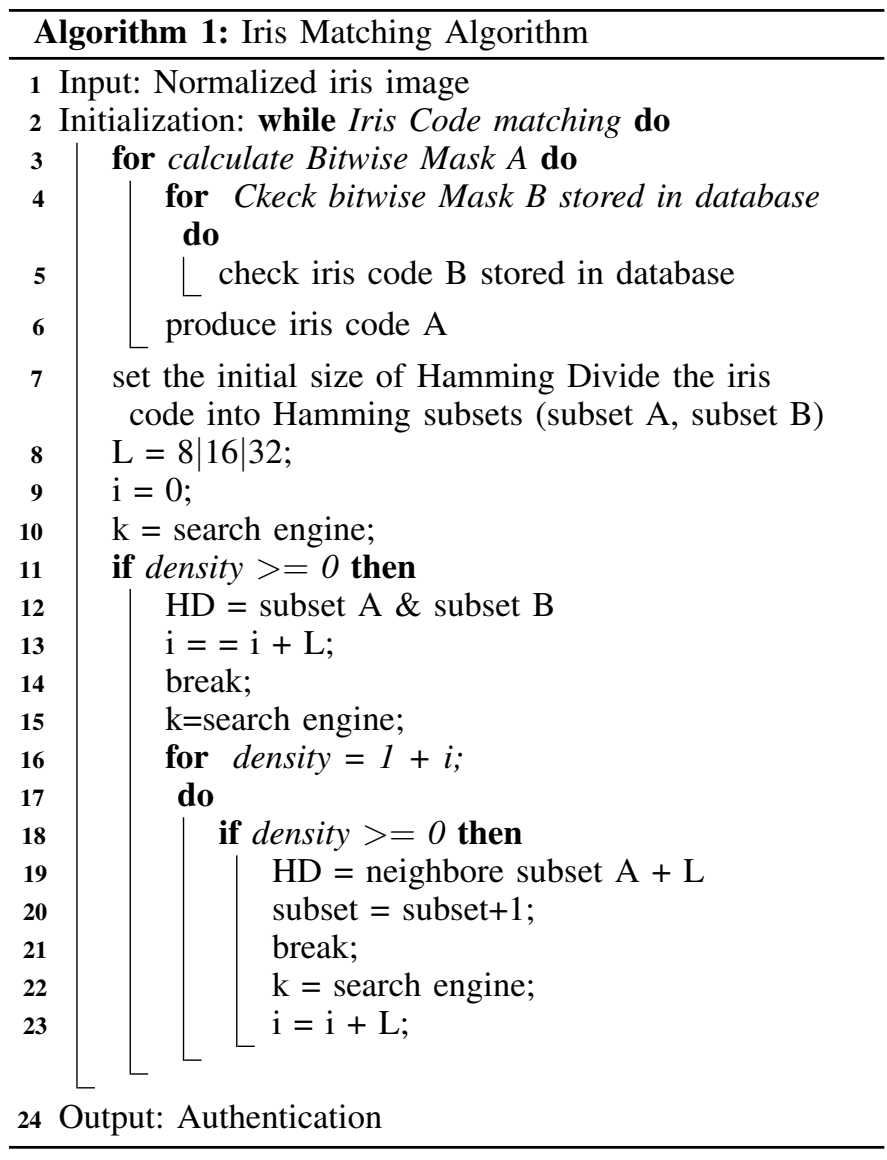

In order to calculated Adaptie Hamming Distance, First, bitwise iris code based Hamming space is divided into Hamming subsets. The initial constant length of Hamming subsets can be the size of 8,16 or 32 bits [42], [43]. Then both iris codes is divided into Hamming subsets with initialization size of $L$. The density value of the masked bit in the Hamming subset can be calculated calculate which is calculated as follows

$$
\operatorname{Mask}(i, j)= \begin{cases}1 & \text { correct bit } \\ 0 & \text { trival bit }\end{cases}
$$

Here the vector Mask represents the trivial bit location in the both iris codes. Where Mask $(i, j)=0$ represents Mask bit, which is excluded from computation and $\operatorname{Mask}(i, j)=1$ correct bit to be compared.

The number of trivial bits are presented in hamming subset as:

$$
P_{(i, j)}=L-\sum_{m=1}^{L} \operatorname{Mask}(i+m, j)
$$

Where hamming subset is equal to $\frac{L}{2}$ or less than adaptive hamming subset. The HD is calculated from the one subset to the neighbor subset, it will indicate that the trivial bits in Hamming subset are larger and the HD is not accurate if it is more than $\frac{L}{2}$. In this step, the search engine verify the density of mask bits in the neighbor hamming subset. If density is low that means the subsets are not affected by noise. Hence, the hamming subsets can expand to the correct bits and these bits will be added to left or right neighbor subset. The location of masked bits can be estimated as follows

$$
\begin{aligned}
\operatorname{Left}_{(i, j)} & =L-\sum_{m=1}^{\frac{L}{2}} \operatorname{Mask}(i+m, j) \\
\operatorname{Right}_{(i, j)} & =L-\sum_{m=\frac{L}{2}}^{L} \operatorname{Mask}(i+m, j)
\end{aligned}
$$

The correct bits will be added to the subset at the left or right to next hamming subset and will be increased as

$$
\begin{gathered}
\operatorname{Left}_{(i, j)} \Longrightarrow L \leftarrow L+\operatorname{Left}(i, j) \\
\operatorname{Right}_{(i, j)} \Longrightarrow L \leftarrow L+\operatorname{Right}(i, j)
\end{gathered}
$$

Hence, the computed Hamming Distance at correspondent bit of subset will be updated by the addition of the correct bits to left neighbor hamming subset, .

\section{EXPERIMENTAL RESUlTS AND DisCUSSION}

\section{A. Dataset}

Experiments are performed on the CASIA Iris database v1, which contains 756 iris images of 108 different people. Totally, $756 * 755 / 2=285390$ pairs of comparisons for each algorithm, 2268 for Intra-Class comparisons and 283122 for Inter-Class comparisons. Which contains 9600 template bits and also for mask bits, respectively.

For the evaluation of our algorithm, we employ False Acceptance Rate (FAR), False Rejection Rate (FRR), Equal Error Rate (EER) and Receiver Operating Curves (ROC). 
In contrast to the degree of match, two error rates FRR and FAR are calculated for selecting a proper point as a threshold for different Adaptive HD using the formula as in equation (13) and equation (14).

$$
\begin{aligned}
F A R & =\frac{\text { No. of time of different imposter }}{\text { No. of comparisons total }} \\
F R R & =\frac{\text { No. of time of different genuine }}{\text { No. of comparisons total }}
\end{aligned}
$$

The error rate can be minimized by selecting the threshold value on the intersection of these two error rates curves as shown in Fig. 8 with EER $=0.004$. If Hamming Distance is smaller then FAR will reduces and FRR increase, FAR will be increased and FRR will be decreased if HD increases.

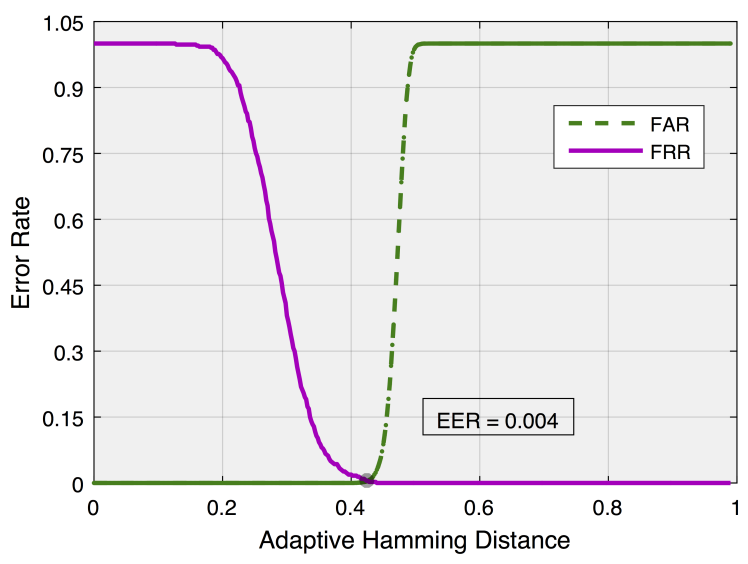

Fig. 8. The Equal Error Rate Value where FAR and FRR are equal.

Fig. 9 shows comparisons of Receiver Operating Curves (ROC) and recognition rate.

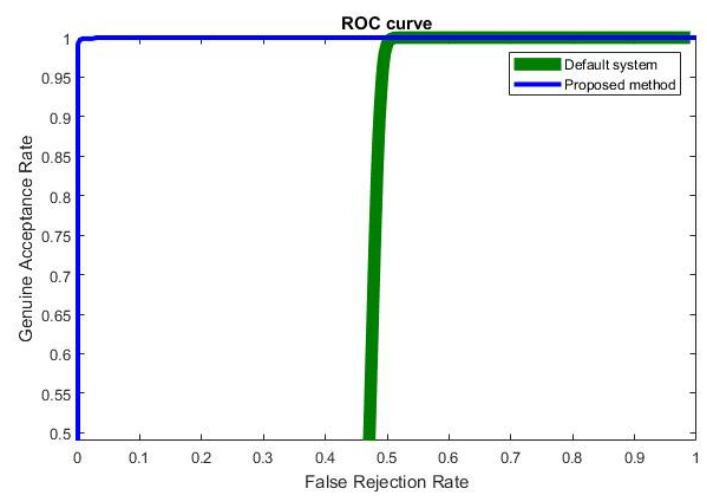

Fig. 9. ROC curves for the system for different recognition rate.

The Table I shows the comparisons performance of the proposed method in terms of recognition rate, False Reject Rate (FRR). The recognition rate of $99.92 \%$ and $\mathrm{FRR}=0.00$ shows the success of method.

\section{B. Intra-Class Comparisons}

In our work 2268 Intra-Class comparisons of iris templates were successfully performed and their Adaptive Hamming Distance distribution is shown in Fig. 10.

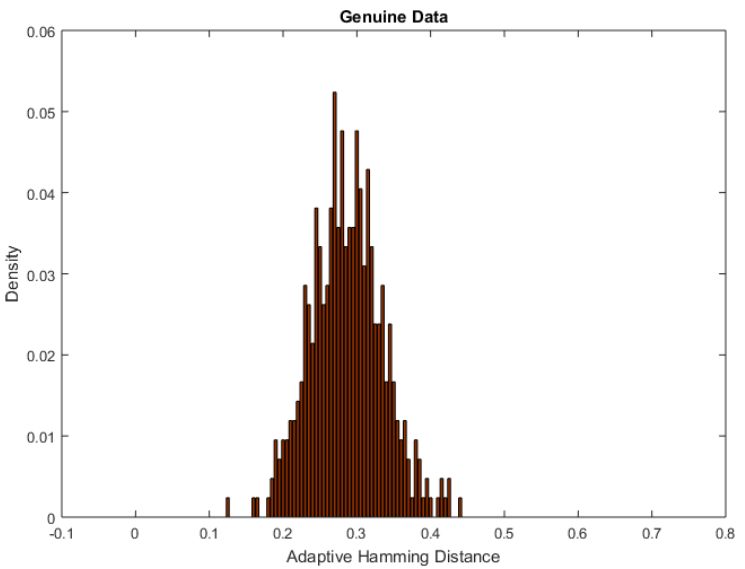

Fig. 10. Intra-Class Adaptive Hamming Distance distribution.

\section{Inter-Class Comparisons}

On our work 283122 Inter-Class comparisons of iris templates were executed successfully and the histogram of distribution is shown in Fig. 11.

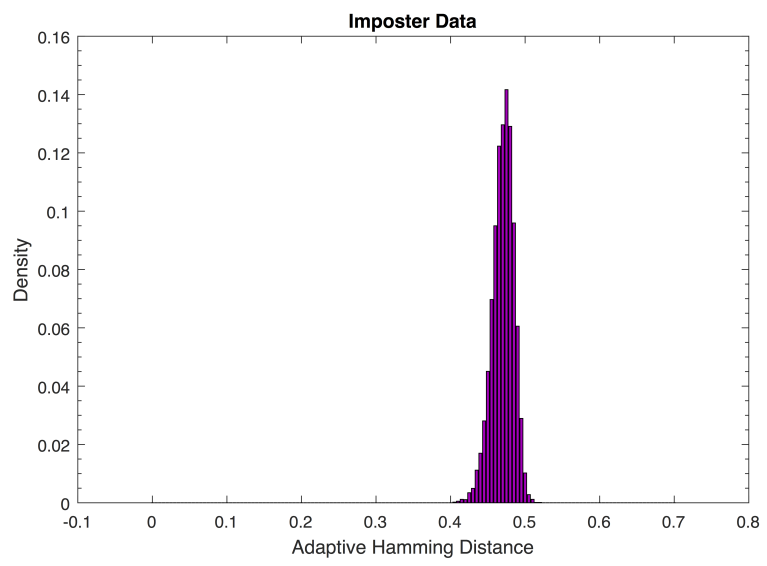

Fig. 11. Inter-Class Adaptive Hamming Distance distribution.

\section{Intra-Class and Inter-Class comparisons}

In the Intra-Class and Inter-Class comparisons very few values were seen that overlap each others. Fig. 12 shows the combined Adaptive HD distribution comparison for $756 * 755 / 2=285390$ pairs.

\section{CONCLUSION}

In our proposed work, Adaptive Hamming Distance is used with the 1D Log-Gabor Filter. Adaptive HD can expand left or right based on the masked bits density. Search engine process the neighboring subsets if the Hamming subset reaches to threshold. Adaptive behavior can give more efficient results than simple HD. To produce a bit-wise biometric template, the normalized iris regions are convolved with the 1D LogGabor filter. Results of the proposed method on CASIA V1.0 show $99.92 \%$ best performance methods with $0 \%$ the state of the art iris recognition FRR, an EER down to $0.004 \%$ 
TABLE I. QUANTITATIVE COMPARISION OF RESUlTS

\begin{tabular}{|c|c|c|c|c|c|c|c|c|}
\hline Methods & Database & Matching Process & Feature Extraction & FAR & FRR & EER & $\begin{array}{c}\text { Overall } \\
\text { Accuracy }\end{array}$ & $\begin{array}{c}\text { Correct Regnition } \\
\text { Rate }\end{array}$ \\
\hline Masek [29] & Dataset & HD with XOR & 2D Gabor & 0.005 & 0.238 & 0.35 & 99.75 & - \\
\hline Avila [32] & Database & $\mathrm{HD}$ & Zero-Crossing & 0.03 & 2.08 & 0.21 & 97.89 & - \\
\hline Soliman et al., [36] & CASIA-V3 CASIA-V1 & HD with XOR & 1-D Log-Gaborwavelets & - & - & - & 98.80 & - \\
\hline Dehkordi et al., [37] & CASIA-V3 & Adaptive HD with XOR & 2-D Log-Gabor wavelets & - & 0.06 & - & - & 99.96 \\
\hline Proposed & CASIA-V1 & Adaptive HD with XOR & 1-D Log-Gabor Filter & 0.08 & 0.00 & 0.004 & 99.92 & 99.996 \\
\hline
\end{tabular}

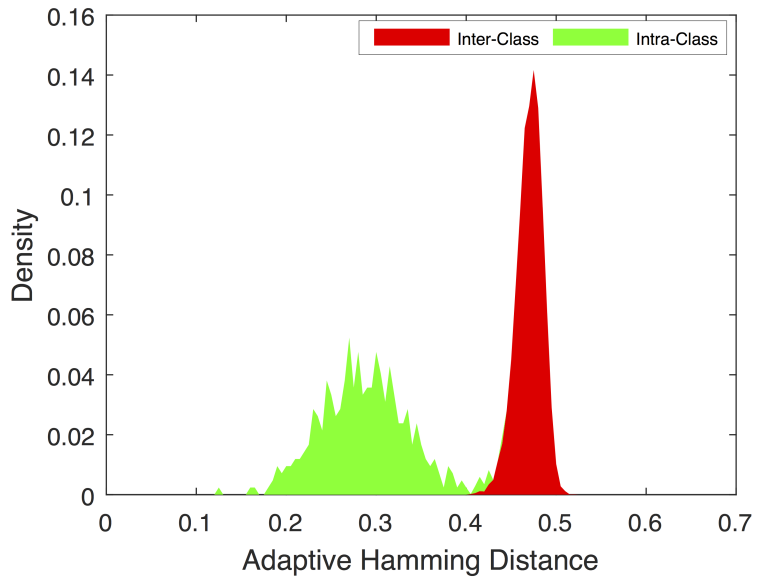

Fig. 12. Intra-Class and Inter-Class Adaptive Hamming Distance distribution.

and with different class of variations i.e. Inter-Class Adaptive HD distribution and Intra-Class Adaptive HD distribution. In future, PCA and FLDA can be incorporated with this model to get better results.

\section{ACKNOWLEDGMENT}

The work presented in this paper was supported by the National Natural Science Foundation of China under Grant no. 61370201

\section{REFERENCES}

[1] N.P. Joseph, "Challenges and Opportunities. Washington," In Biometric Recognition; I.M. Lynette, Eds.; DC: The National Academies Press: Washington, 2010. [CrossRef]

[2] L. Ma, Y. Wang and T. Tan, "Iris Recognition Based on Multichannel Gabor Filtering," ACCV2002: The 5th Asian Conference on Computer Vision, pp. 23-25, January 2002.

[3] J. Daugman, "How Iris Recognition Works," IEEE Transactions on Circuits and Systems for Video Technology, pp. 21-30, 2004. [CrossRef]

[4] J. Daugman, "Probing the Uniqueness and Randomness of IrisCodes: Results From 200 Billion Iris Pair Comparisons," Proceedings of the IEEE, November 2006. [CrossRef]

[5] R. P. Wildes, "Iris recognition: an emerging biometric technology," Proceedings of the IEEE, pp. 1348-1363, September 1997. [CrossRef]

[6] W. W. Boles, B. A. Boashash, "Human identification technique using images of the iris and wavelet transform," IEEE Transactions on Signal Processing, pp. 1185-1188, 1998. [CrossRef]
[7] Z. Yong, T. Tieniu and W. YunHong, "Biometric personal identification based on iris patterns," Proceedings 15th International Conference on Pattern Recognition. ICPR-2000, Espagne, 2000. [CrossRef]

[8] S. L. Lim, K.L. Lee, O. B. Byeon, et al., "Efficient Iris Recognition through Improvement of Feature Vector and Classifier," ETRI Journal, pp. 61-70, 2001. [CrossRef]

[9] T. Christel-Loic, M. Lionel, T. Lionel, et al., "Person Identification Technique Using Human Iris Recognition," The 15th International Conference on Vision Interface, pp. 294-299, 2002.

[10] E. Rydgren, T. Ea, F. Amiel, et al., "Person Identification Technique Using Human Iris Recognition," IEEE International Conference on Image Processing (ICIP'04), Singapore, 2004.

[11] S. Noh, K. Bae, K. Park, et al., "A New Iris Recognition Method Using Independent Component Analysis," IEICE Transactions on Information and Systems, pp. 2573-2581, 2005.

[12] J. Daugman, "New Methods in Iris Recognition," IEEE Transactions on Systems, Man and Cybernetics, Part B (Cybernetics), pp. 1167-1175, 2007. [CrossRef]

[13] M. Nabti, L. Ghouti and A. Bouridane, "New An effective and fast iris recognition system based on a combined multiscale feature extraction technique," Pattern Recognition, pp. 868-879 2008. [CrossRef]

[14] M. Lionel, "from the pixel to the integrated iris recognition system," University Paul Cezanne (Aix-Marseille III), Marseille, 2008.

[15] T. Wang, M. Han, H. Wan, et al., "A robust and fast eyelash detection basted on expectation maximization and Gaussian mixture model," Springer, pp. 9397, 2011. [CrossRef]

[16] A. Walid, K. Lotfi and M. Nouri, "A fast and accurate eyelids and eyelashes detection approach for iris segmentation," International conference on IEEE, Sousse, Tunisia, June 2013. [CrossRef]

[17] Z. He, T. Tan, Z. Sun, et al., "Toward accurate and fast iris segmentation for iris biometrics," IEEE Transactions, pp. 1670-1684, 2009. [CrossRef]

[18] Y. Hu, K. Sirlantzis and G. Howells, "Improving color iris segmentation using a model selection technique," Pattern Recognition, pp.24-32, 2015. [CrossRef]

[19] D. S. Swati, C. RajaBhushnam, "Review of IRIS recognition techniques" 2017 International Conference on Algorithms, Methodology, Models and Applications in Emerging Technologies (ICAMMAET), Chennai, India, Feb 2017. [CrossRef]

[20] A. M. A. Musab, M. T. Nooritawati, "Cancelable biometrics technique for iris recognition," 2018 IEEE Symposium on Computer Applications \& Industrial Electronics (ISCAIE), Penang, Malaysia, April 2018. [CrossRef]

[21] J. Daugman, "High confidence visual recognition of persons by a test of statistical independence," IEEE Transactions on Pattern Analysis and Machine Intelligence, pp. 1148-1161, November 1993. [CrossRef]

[22] Z. Sun, T. Tan, "Ordinal measures for iris recognition," IEEE Transaction, pp. 2211-2226, 2009. [CrossRef]

[23] L. Ma, T. Tan, Y. Wang, et al., "Efficient Iris Recognition by Characterizing Key Local Variations," IEEE Transactions on Image Processing, pp. 739-750, 2004. [CrossRef] 
[24] C. W. Tan, A. Kumar, "Accurate iris recognition at a distance using stabilized iris encoding and Zernike moments phase features," IEEE Transaction, pp. 3962-3974, 2014. [CrossRef]

[25] K. P. Hollingsworth, K. W. Bowyer and P. J. Flynn, "The best bits in an iris code," IEEE Transaction, pp. 964-973, 2009. [CrossRef]

[26] Y. Hu, K. Sirlantzis and G. Howells, "Exploiting stable and discriminative iris weight map for iris recognition under less constrained environment," IEEE International Conference on Biometrics, Theory, Arlington, VA, USA, September 2015. [CrossRef]

[27] C. W. Tan, A. Kumar, "Efficient and accurate at a distance iris recognition using geometric key-based iris encoding," IEEE Transaction, pp. 1518-1526, 2014. [CrossRef]

[28] P. Li, G. Wu, "Iris recognition using ordinal encoding of log-Euclidean covariance matrices," IEEE International Conference on Pattern Recognition, Tsukuba, Japan, November 2012.

[29] L. Masek, "Recognition of Human Iris Patterns for Biometric Identification," University of Western Australia, Australia, 2002.

[30] S. Shah, A. Ross, "Iris segmentation using geodesic active contours," IEEE Signal Processing Society, pp. 824-836, 2009. [CrossRef]

[31] S.Dey, D. Samanta, "A novel approach to iris localization for iris biometric processing," International Journal of Biological and Medical Sciences, 2008.

[32] C. Sanchez-Avila, R. Sanchez-Reillo and D. De Martin-Roche, "Iris recognition for biometric identification using dyadic wavelet transform zero-crossing," IEEE 35th International Carnahan Conference on Security Technology, Quebec City, London, England, UK, October 2001.[CrossRef]

[33] L. Ma, Y. Wang and T. Tan, "Iris Recognition Based on Multichannel Gabor Filtering," Proc. The 5th Asian Conference on Computer Vision,
January 2002.

[34] T. Christel-Loic, M. Lionel, T. Lionel, et al., "Person Identification Technique Using Human Iris Recognition," The 15th International Conference on Vision Interface, 2002.

[35] H. Rai, A. Yadav, "Iris recognition using combined support vector machine and Hamming distance approach," Expert Systems with Applications, pp. 588-593, 2014. [CrossRef]

[36] N. F. Soliman, E. Mohamed and F. Magdi, "Efficient iris localization and recognition," Optik-International Journal for Light and Electron Optics, pp. 469-475, 2017. [CrossRef]

[37] A. B. Dehkordi, S. A. R. Abu-Bakar, "Iris code matching using adaptive Hamming distance," 2015 IEEE International Conference on Signal and Image Processing Applications (ICSIPA), Malaysia, 2015. [CrossRef]

[38] CASIA V.1.0 iris image database version one. http://www.cbsr.ia.ac.cn

[39] P. Yao, J. Li, X. Ye, et al., "Iris recognition algorithm using modified log-Gabor filters," International Conferenece on Pattern Recognition, Hong Kong, China, August 2006. [CrossRef]

[40] L. Masek, v. Kovesi, "MATLAB Source Code for a Biometric Identification System Based on Iris Patterns," School of Computer Science and Software Engineering, University of Western Australia, 2003. http: //www.csse.uwa.edu.au/ pk/studentprojects/libor/sourcecode.html6

[41] A. Ross, D. Nandakumar and A. K. Jain, "Biometric personal identification system based on iris analysis," in Handbook of Multibiometrics, Springer, Heidelberg, 2006.

[42] K. W. Bowyer, K. P. Hollingsworth and P. J. Flynn, "Handbook of iris recognition," A survey of iris biometrics research, 2008-2010, Springer, pp. 15-54, 2013.

[43] K. W. Bowyer, "The results of the NICE.II Iris biometrics competition," Pattern Recognition Letters, pp. 965-969, 2012. [CrossRef] 